\title{
Global existence and stability results for a nonlinear Timoshenko system of thermoelasticity of type III with delay
}

Jianghao Hao* (10) and Jing Wei

"Correspondence:

hjhao@sxu.edu.cn

School of Mathematical Sciences,

Shanxi University, Taiyuan, China

\section{Springer}

\begin{abstract}
In this paper, we consider a nonlinear thermoelastic system of Timoshenko type with delay. It is known that an arbitrarily small delay may be the source of instability. The delay term works on the second equation which describes the motion of a rotation angle. We establish the well-posedness and the stability of the system for the cases of equal and nonequal speeds of wave propagation. Our results show that the damping effect is strong enough to uniformly stabilize the system even in the presence of time delay under suitable conditions by using perturbed energy functional technique and improve the related results.
\end{abstract}

MSC: 35L70; 35L75; 93D20

Keywords: Timoshenko system of thermoelasticity of type III; Delay term; Well-posedness; Stability

\section{Introduction}

In this paper, we consider a nonlinear Timoshenko-type system of thermoelasticity of type III with delay:

$$
\begin{cases}\rho_{1} \phi_{t t}-K\left(\phi_{x}+\psi\right)_{x}=0, & (x, t) \in(0,1) \times(0, \infty), \\ \rho_{2} \psi_{t t}-b \psi_{x x}+K\left(\phi_{x}+\psi\right)+\beta \theta_{t x} & \\ \quad+\mu_{1} \psi_{t}(x, t)+\mu_{2} \psi_{t}(x, t-\tau)+f(\psi)=0, & (x, t) \in(0,1) \times(0, \infty), \\ \rho_{3} \theta_{t t}-\delta \theta_{x x}+\gamma \psi_{t x}-k \theta_{t x x}=0, & (x, t) \in(0,1) \times(0, \infty),\end{cases}
$$

in which $\rho_{1}, \rho_{2}, \rho_{3}, K, b, k, \beta, \gamma, \delta, \mu_{1}, \mu_{2}, \tau$ are positive constants. In this system, $\mu_{1} \psi_{t}$ represents a frictional damping, $\mu_{2} \psi_{t}(x, t-\tau)$ represents a delay term, and $f(\psi)$ is a forcing term. We impose the following initial and boundary conditions:

$$
\left\{\begin{array}{lll}
\psi(x, 0)=\psi_{0}(x), & \psi_{t}(x, 0)=\psi_{1}(x), & x \in(0,1), \\
\phi(x, 0)=\phi_{0}(x), & \phi_{t}(x, 0)=\phi_{1}(x), & x \in(0,1), \\
\theta(x, 0)=\theta_{0}(x), \quad \theta_{t}(x, 0)=\theta_{1}(x), & x \in(0,1), \\
\phi(0, t)=\phi(1, t)=\psi(0, t)=\psi(1, t) & \\
=\theta_{x}(0, t)=\theta_{x}(1, t)=0, & t \in(0, \infty), \\
\psi_{t}(x, t-\tau)=f_{0}(x, t-\tau), & (x, t) \in(0,1) \times(0, \tau) .
\end{array}\right.
$$

(C) The Author(s) 2018. This article is distributed under the terms of the Creative Commons Attribution 4.0 International License (http://creativecommons.org/licenses/by/4.0/), which permits unrestricted use, distribution, and reproduction in any medium, provided you give appropriate credit to the original author(s) and the source, provide a link to the Creative Commons license, and indicate if changes were made. 
We start our literature review with the pioneer work of Messaoudi and Said-Houari [1]. The authors considered the system as follows:

$$
\begin{cases}\rho_{1} \phi_{t t}-K\left(\phi_{x}+\psi\right)_{x}=0, & (x, t) \in(0,1) \times(0, \infty), \\ \rho_{2} \psi_{t t}-b \psi_{x x}+K\left(\phi_{x}+\psi\right)+\beta \theta_{t x}=0, & (x, t) \in(0,1) \times(0, \infty), \\ \rho_{3} \theta_{t t}-\delta \theta_{x x}+\gamma \psi_{t x}-k \theta_{t x x}=0, & (x, t) \in(0,1) \times(0, \infty)\end{cases}
$$

They obtained an exponential decay result under equal wave speeds $\left(\frac{K}{\rho_{1}}=\frac{b}{\rho_{2}}\right)$. Later Messaoudi and Fareh [2] studied the case of nonequal speeds $\left(\frac{K}{\rho_{1}} \neq \frac{b}{\rho_{2}}\right)$ and established a polynomial decay result. In [3], the author consider a vibrating nonlinear Timoshenko system with thermoelasticity with second sound as follows:

$$
\begin{cases}\rho_{1} \phi_{t t}-K\left(\phi_{x}+\psi\right)_{x}=0, & (x, t) \in(0,1) \times(0, \infty), \\ \rho_{2} \psi_{t t}-b \psi_{x x}+K\left(\phi_{x}+\psi\right)+\delta \theta_{x}+\alpha(t) h\left(\psi_{t}\right)=0, & (x, t) \in(0,1) \times(0, \infty), \\ \rho_{3} \theta_{t}+q_{x}+\gamma \psi_{t x}=0, & (x, t) \in(0,1) \times(0, \infty), \\ \tau q_{t}+\beta q+\theta_{x}=0, & (x, t) \in(0,1) \times(0, \infty)\end{cases}
$$

They established general decay results for the cases of $\mu=0$ and $\mu \neq 0$ with the constant $\mu=\left(\tau-\frac{\rho_{1}}{K \rho_{3}}\right)\left(\frac{\rho_{2}}{b}-\frac{\rho_{1}}{K}\right)-\frac{\tau \delta^{2} \rho_{1}}{b K \rho_{3}}$.

On the other hand, Timoshenko systems with delay term have attracted extensive attention, and the increasing complexity of their types makes research more significant. An arbitrarily small delay may be the source of instability, see [4, 5]. Racke [6] considered the following coupled system of linear thermoelastic equations with constant delays $\tau_{1}$ and $\tau_{2}$ :

$$
\begin{cases}a v_{t t}-d v_{x x}\left(x, t-\tau_{1}\right)+\beta \theta_{x}=0, & (x, t) \in(0, L) \times(0, \infty) \\ b \theta_{t}-k \theta_{x x}\left(x, t-\tau_{2}\right)+\beta v_{x t}=0, & (x, t) \in(0, L) \times(0, \infty) \\ v(0, t)=v(L, t)=\theta_{x}(0, t)=\theta_{x}(L, t)=0, & t \in(0, \infty)\end{cases}
$$

He obtained that the solution of problem (1.4) is instable with any delay $\tau_{1}>0$ or $\tau_{2}>0$. In recent years constant delay $\tau$ has been extended to the time-varying function $\tau(t)$ in the thermoelastic equations, see [7]. Also, in [8] Nicaise and Pignotti studied the initialboundary value problem of wave equation with boundary distributed delay as follows:

$$
\begin{cases}u_{t t}-\Delta u=0, & (x, t) \in \Omega \times(0, \infty), \\ u=0, & (x, t) \in \Gamma_{0} \times(0, \infty), \\ \frac{\partial u}{\partial v}(t)+\int_{\tau_{1}}^{\tau_{2}} \mu(s) u_{t}(t-s) d s+\mu_{0} u_{t}(t)=0, & (x, t) \in \Gamma_{1} \times(0, \infty), \\ u(x, 0)=u_{0}(x), \quad u_{t}(x)=u_{1}(x), & x \in \Omega, \\ u_{t}(x,-t)=f_{0}(x,-t), & (x, t) \in \Gamma_{1} \times\left(0, \tau_{2}\right) .\end{cases}
$$

They proved an exponential stability result for system (1.5) with the condition

$$
\int_{\tau_{1}}^{\tau_{2}} \mu(s) d s<\mu_{0}
$$

and when the boundary distributed delay term in the above system is replaced by the internal feedback $\int_{\tau_{1}}^{\tau_{2}} a(x) \mu(s) u_{t}(t-s) d s$ with $a(x)$ satisfying some suitable conditions. They 
also obtained that the energy of solution is exponentially decaying to zero under the condition

$$
\|a\|_{\infty} \int_{\tau_{1}}^{\tau_{2}} \mu(s) u_{t}(t-s) d s<\mu_{0} .
$$

We refer the reader to [9-14] for more analogous results. Kafini et al. [15], considered a one-dimensional Timoshenko-type system

$$
\begin{cases}\rho_{1} \phi_{t t}-K\left(\phi_{x}+\psi\right)_{x}+\mu_{1} \psi_{t}(x, t)+\mu_{2} \psi_{t}(x, t-\tau)=0, & (x, t) \in(0,1) \times(0, \infty), \\ \rho_{2} \psi_{t t}-b \psi_{x x}+K\left(\phi_{x}+\psi\right)+\beta \theta_{t x}=0, & (x, t) \in(0,1) \times(0, \infty), \\ \rho_{3} \theta_{t t}-\delta \theta_{x x}+\gamma \psi_{t x}-k \theta_{t x x}=0, & (x, t) \in(0,1) \times(0, \infty) .\end{cases}
$$

They proved the well-posedness of system (1.6) and established an exponential decay result under the condition $\frac{K}{\rho_{1}}=\frac{b}{\rho_{2}}$ and a polynomial decay result under the condition $\frac{K}{\rho_{1}} \neq \frac{b}{\rho_{2}}$. For a Timoshenko system with time delay and forcing term at the same time

$$
\begin{cases}\rho_{1} \varphi_{t t}-K\left(\varphi_{x}+\psi\right)_{x}=0, & (x, t) \in(0,1) \times(0, \infty), \\ \rho_{2} \psi_{t t}-b \psi_{x x}+K\left(\varphi_{x}+\psi\right) & \\ +\mu_{1} \psi_{t}(x, t)+\mu_{2} \psi_{t}(x, t-\tau)+f(\psi)=0, & (x, t) \in(0,1) \times(0, \infty),\end{cases}
$$

Feng and Pelicer [16] obtained an exponential stability under equal wave speeds. In the present paper, when $\frac{K}{\rho_{1}}=\frac{b}{\rho_{2}}$, we extend their result to nonlinear Timoshenko system of thermoelasticity of type III by using the perturbed energy functional technique as well, and when $\frac{K}{\rho_{1}} \neq \frac{b}{\rho_{2}}$, we achieve a polynomial decay estimate.

This paper is organized as follows. In Sect. 2, we present some assumptions and preliminary works. In Sect. 3, we establish the well-posedness of system (1.1)-(1.2) by using semigroup theory in $[15,16]$. In Sect. 4 , we prove the decay results in two cases by using energy methods.

\section{Preliminaries}

In this section, we present some materials needed for our main results. For simplicity of notations, hereafter we denote by $\|\cdot\|_{q}$ the Lebesgue space $L^{q}(\Omega)$ norm, and by $\|\cdot\|$ the Lebesgue space $L^{2}(\Omega)$ norm.

Assumption 2.1 Assume that $f: \mathbb{R} \rightarrow \mathbb{R}$ with $f(0)=0$ satisfies

$$
\left|f\left(\psi^{1}\right)-f\left(\psi^{2}\right)\right| \leq k_{0}\left(\left|\psi^{1}\right|^{\varsigma}+\left|\psi^{2}\right|^{\varsigma}\right)\left|\psi^{1}-\psi^{2}\right|, \quad \psi^{1}, \psi^{2} \in \mathbb{R},
$$

where $k_{0}>0, \varsigma \geq 1$ are constants such that

$$
|f(\psi)| \leq k_{0}|\psi|^{\varsigma}|\psi|, \quad \psi \in \mathbb{R} .
$$

In addition we assume that

$$
0 \leq \hat{f}(\psi) \leq f(\psi) \psi, \quad \psi \in \mathbb{R}
$$

in which $\hat{f}(\psi):=\int_{0}^{\psi} f(s) d s$. 
In order to deal with the delay term, we define the following new variable:

$$
z(x, \rho, t):=\psi_{t}(x, t-\tau \rho), \quad x \in(0,1), \rho \in(0,1), t>0 .
$$

Thus we have

$$
\tau z_{t}(x, \rho, t)+z_{\rho}(x, \rho, t)=0, \quad \rho \in(0,1), t>0 .
$$

Then system (1.1)-(1.2) is transformed to

$$
\begin{cases}\rho_{1} \phi_{t t}-K\left(\phi_{x}+\psi\right)_{x}=0, & (x, t) \in(0,1) \times(0, \infty), \\ \rho_{2} \psi_{t t}-b \psi_{x x}+K\left(\phi_{x}+\psi\right)+\beta \theta_{t x} & \\ \quad+\mu_{1} \psi_{t}(x, t)+\mu_{2} z(x, 1, t)+f(\psi)=0, & (x, t) \in(0,1) \times(0, \infty), \\ \rho_{3} \theta_{t t}-\delta \theta_{x x}+\gamma \psi_{t x}-k \theta_{t x x}=0, & (x, t) \in(0,1) \times(0, \infty), \\ \tau z_{t}(x, \rho, t)+z_{\rho}(x, \rho, t)=0, & (x, \rho, t) \in(0,1) \times(0,1) \times(0, \infty), \\ \psi(x, 0)=\psi_{0}(x), \quad \psi_{t}(x, 0)=\psi_{1}(x), & \\ z(x, 0, t)=\psi_{t}(x, t), & x \in(0,1), \\ \phi(x, 0)=\phi_{0}(x), \quad \phi_{t}(x, 0)=\phi_{1}(x), & x \in(0,1), \\ \theta(x, 0)=\theta_{0}(x), \quad \theta_{t}(x, 0)=\theta_{1}(x), & x \in(0,1), \\ \phi(0, t)=\phi(1, t)=\psi(0, t)=\psi(1, t) & \\ \quad=\theta_{x}(0, t)=\theta_{x}(1, t)=0, & t \in(0, \infty), \\ z(x, \rho, 0)=f_{0}(x,-\rho \tau), & (x, \rho) \in(0,1) \times(0,1) .\end{cases}
$$

In order to use the Poincaré inequality for $\theta$, as in [15], we introduce

$$
\bar{\theta}(x, t):=\theta(x, t)-t \int_{0}^{1} \theta_{1}(x) d x-\int_{0}^{1} \theta_{0}(x) d x .
$$

Then by $(2.4)_{3}$ we have

$$
\int_{0}^{1} \bar{\theta}(x, t) d x=0, \quad t \geq 0
$$

After a simple substitution, we see that $(\phi, \psi, \bar{\theta}, z)$ satisfies (2.4). From now on, we work with $\bar{\theta}$ but write $\theta$ for convenience.

\section{Well-posedness result}

In this section, we shall investigate the well-posedness of problem (2.1) with semigroup theory, we start with the vector function $\mathcal{U}(t)=(\phi, \varphi, \psi, u, \theta, v, z)^{T}$, where $\varphi=\phi_{t}, u=\psi_{t}$, and $v=\theta_{t}$. We introduce as in [15]

$$
\begin{aligned}
& L_{\star}^{2}(0,1):=\left\{\omega \in L^{2}(0,1) \mid \int_{0}^{1} \omega(s) d s=0\right\}, \\
& H_{\star}^{1}(0,1):=H^{1}(0,1) \cap L_{\star}^{2}(0,1), \\
& H_{\star}^{2}(0,1):=\left\{\omega \in H^{2}(0,1) \mid \omega_{x}(0)=\omega_{x}(1)=0\right\} .
\end{aligned}
$$


Then we define the energy space by

$$
\mathcal{H}:=H_{0}^{1}(0,1) \times L^{2}(0,1) \times H_{0}^{1}(0,1) \times L^{2}(0,1) \times H_{\star}^{1}(0,1) \times L_{\star}^{2}(0,1) \times L^{2}\left((0,1), L^{2}(0,1)\right),
$$

equipped with the following inner product:

$$
\begin{aligned}
\langle\mathcal{U}, \tilde{\mathcal{U}}\rangle_{\mathcal{H}}:= & \gamma \int_{0}^{1}\left[\rho_{1} \varphi \tilde{\varphi}+\rho_{2} u \tilde{u}+K\left(\phi_{x}+\psi\right)\left(\widetilde{\phi}_{x}+\widetilde{\psi}\right)+b \psi_{x} \widetilde{\psi}_{x}\right] d x \\
& +\beta \int_{0}^{1}\left(\rho_{3} v \widetilde{v}+\delta \theta_{x} \widetilde{\theta}_{x}\right) d x+\xi \int_{0}^{1} \int_{0}^{1} z(x, \rho) \widetilde{z}(x, \rho) d \rho d x
\end{aligned}
$$

in which $\xi$ is a positive constant satisfying

$$
\gamma \tau \mu_{2} \leq \xi \leq \gamma \tau\left(2 \mu_{1}-\mu_{2}\right)
$$

Thus system (2.4) can be re-written as

$$
\left\{\begin{array}{l}
\frac{d}{d t} \mathcal{U}(t)+\mathcal{A} \mathcal{U}(t)=\mathcal{F}(\mathcal{U}) \\
\mathcal{U}(0)=\mathcal{U}_{0}=\left(\phi_{0}, \phi_{1}, \psi_{0}, \psi_{1}, \theta_{0}, \theta_{1}, f_{0}(\cdot,-\tau \rho)\right)^{T}
\end{array}\right.
$$

where the operators $\mathcal{A}$ and $\mathcal{F}$ are defined by

$$
\mathcal{A} \mathcal{U}:=\left(\begin{array}{c}
-\varphi \\
-\frac{K}{\rho_{1}}\left(\phi_{x}+\psi\right)_{x} \\
-u \\
-\frac{b}{\rho_{2}} \psi_{x x}+\frac{K}{\rho_{2}}\left(\phi_{x}+\psi\right)+\frac{\beta}{\rho_{2}} v_{x}+\frac{\mu_{1}}{\rho_{2}} u+\frac{\mu_{2}}{\rho_{2}} z(x, 1, t) \\
-v \\
-\frac{\delta}{\rho_{3}} \theta_{x x}+\frac{\gamma}{\rho_{3}} u_{x}-\frac{k}{\rho_{3}} v_{x x} \\
\frac{1}{\tau} z_{\rho}
\end{array}\right), \quad \mathcal{F}(\mathcal{U}):=\left(\begin{array}{c}
0 \\
0 \\
0 \\
-\frac{1}{\rho_{2}} f(\psi) \\
0 \\
0 \\
0
\end{array}\right)
$$

with

$$
D(\mathcal{A})=\left\{\begin{array}{l}
\mathcal{U} \in \mathcal{H} \mid \phi, \psi \in H^{2}(0,1) \cap H_{0}^{1}(0,1), \theta, v \in H_{\star}^{1}(0,1), \varphi, u \in H_{0}^{1}(0,1), \\
\delta \theta+k v \in H_{\star}^{2}(0,1), z, z_{\rho} \in L^{2}\left((0,1), L^{2}(0,1)\right), z(x, 0)=\psi(x)
\end{array}\right\}
$$

and the initial value $\left(\theta_{0}, \theta_{1}\right)$ satisfies

$$
\left\{\begin{array}{l}
\theta_{0}:=\theta_{0}(x)-\int_{0}^{1} \theta_{0}(x) d x \\
\theta_{1}:=\theta_{1}(x)-\int_{0}^{1} \theta_{1}(x) d x
\end{array}\right.
$$

By using the same methods as those in [15] and in [16], we can obtain the following Lemmas 3.1 and 3.2, respectively. We omit the proof.

Lemma 3.1 The operator $\mathcal{A}$ defined in (3.3) is the infinitesimal generator of a $C_{0^{-}}$ semigroup in $\mathcal{H}$.

Lemma 3.2 The operator $\mathcal{F}$ is locally Lipschitz in $\mathcal{H}$. 
According to Pazy [17], Chap. 6, we can obtain the existing results as follows. We omit the proof.

Theorem 3.3 Suppose that Assumption 2.1 holds and $\mu_{2} \leq \mu_{1}$. For any initial value $\mathcal{U}_{0} \in$ $\mathcal{H}$, system (3.3) admits a unique solution $\mathcal{U} \in C(0, \infty ; \mathcal{H})$. Moreover, if $\mathcal{U}_{0} \in \mathcal{D}(\mathcal{A})$, system (3.3) admits a unique solution $\mathcal{U} \in C(0, \infty ; \mathcal{D}(\mathcal{A})) \cap C^{1}(0, \infty ; \mathcal{H})$.

We introduce the first order energy of problem (2.4) as

$$
\begin{aligned}
E(t):= & \frac{1}{2} \int_{0}^{1}\left[\gamma\left(\rho_{1} \phi_{t}^{2}+K\left(\phi_{x}+\psi\right)^{2}+\rho_{2} \psi_{t}^{2}+b \psi_{x}^{2}\right)+\beta\left(\rho_{3} \theta_{t}^{2}+\delta \theta_{x}^{2}\right)\right] d x \\
& +\frac{\xi}{2} \int_{0}^{1} \int_{0}^{1} z^{2}(x, \rho, t) d \rho d x+\gamma \int_{0}^{1} \hat{f}(\psi) d x,
\end{aligned}
$$

and the second order energy of problem (2.4) as (if $\mathcal{U}_{0} \in \mathcal{D}(\mathcal{A})$ )

$$
\begin{aligned}
E_{2}(t):= & \frac{1}{2} \int_{0}^{1}\left[\gamma\left(\rho_{1} \phi_{t t}^{2}+K\left(\phi_{t x}+\psi_{t}\right)^{2}+\rho_{2} \psi_{t t}^{2}+b \psi_{t x}^{2}\right)+\beta\left(\rho_{3} \theta_{t t}^{2}+\delta \theta_{t x}^{2}\right)\right] d x \\
& +\frac{\xi}{2} \int_{0}^{1} \int_{0}^{1} z_{t}^{2}(x, \rho, t) d \rho d x+\gamma \int_{0}^{1} \hat{f}\left(\psi_{t}\right) d x .
\end{aligned}
$$

Lemma 3.4 Let $(\phi, \psi, \theta, z)$ be the solution of problem (2.4). Then the energy functional defined by (3.6) satisfies

$$
E^{\prime}(t) \leq-C \int_{0}^{1}\left(\theta_{t x}^{2}+\psi_{t}^{2} d x+z^{2}(x, 1, t)\right) d x \leq 0
$$

with some constant $C \geq 0$.

Proof Multiplying the first three equations in (2.4) by $\gamma \phi_{t}, \gamma \psi_{t}, \beta \theta_{t}$, respectively, and integrating over $(0,1)$, and multiplying $(2.4)_{4}$ by $\frac{\xi z}{\tau}$ and integrating over $(0,1) \times(0,1)$ with respect to $\rho$ and $x$, we get

$$
\begin{aligned}
\frac{1}{2} \frac{d}{d t} \int_{0}^{1}\left[\gamma\left(\rho_{1} \phi_{t}^{2}+K\left(\phi_{x}+\psi\right)^{2}+\rho_{2} \psi_{t}^{2}+\psi_{x}^{2}\right)+\beta\left(\rho_{3} \theta_{t}^{2}+\delta \theta_{x}^{2}\right)\right] d x \\
\quad+\frac{\xi}{2} \frac{d}{d t} \int_{0}^{1} \int_{0}^{1} z^{2}(x, 1, t) d \rho d x+\gamma \frac{d}{d t} \int_{0}^{1} \hat{f}(\psi(t)) d x \\
=-\beta \kappa \int_{0}^{1} \theta_{t x}^{2} d x-\gamma \mu_{1} \int_{0}^{1} \psi_{t}^{2} d x-\frac{\xi}{\tau} \int_{0}^{1} \int_{0}^{1} z z_{\rho}(x, \rho, t) d \rho d x \\
\quad-\gamma \mu_{2} \int_{0}^{1} \psi_{t} z(x, 1, t) d x
\end{aligned}
$$

For the last two terms on the right-hand side, by using Hölder's inequality and Young's inequality, we have

$$
\begin{aligned}
-\frac{\xi}{\tau} \int_{0}^{1} \int_{0}^{1} z z_{\rho}(x, \rho, t) d \rho d x & =-\frac{\xi}{2 \tau} \int_{0}^{1} \int_{0}^{1} \frac{\partial}{\partial \rho} z^{2}(x, \rho, t) d \rho d x \\
& =\frac{\xi}{2 \tau}\left(\int_{0}^{1} \psi_{t}^{2} d x-\int_{0}^{1} z^{2}(x, 1, t) d x\right)
\end{aligned}
$$


and

$$
-\gamma \mu_{2} \int_{0}^{1} \psi_{t} z(x, 1, t) d x \leq \frac{\gamma \mu_{2}}{2}\left(\int_{0}^{1} \psi_{t}^{2} d x+\int_{0}^{1} z^{2}(x, 1, t) d x\right)
$$

Combining (3.9)-(3.11), we obtain

$$
\begin{aligned}
E^{\prime}(t) \leq & -\beta \kappa \int_{0}^{1} \theta_{t x}^{2} d x-\gamma\left(\mu_{1}-\frac{\xi}{2 \tau \gamma}-\frac{\mu_{2}}{2}\right) \int_{0}^{1} \psi_{t}^{2} d x \\
& -\gamma\left(\frac{\xi}{2 \tau \gamma}-\frac{\mu_{2}}{2}\right) \int_{0}^{1} z^{2}(x, 1, t) d x
\end{aligned}
$$

The above assumption (3.2) implies that there exists a constant $C \geq 0$ such that

$$
E^{\prime}(t) \leq-C\left\{\int_{0}^{1} \theta_{t x}^{2} d x+\int_{0}^{1} \psi_{t}^{2} d x+\int_{0}^{1} z^{2}(x, 1, t) d x\right\} \leq 0
$$

This gives (3.8).

\section{Energy decay result}

In this section, we shall state and prove our decay result.

Theorem 4.1 Suppose that Assumption 2.1 holds and $\mu_{1}>\mu_{2}$. For any initial value $\mathcal{U}_{0} \in$ $\mathcal{H}$, there exist positive constants $C$ and $\alpha$ such that the energy of problem (2.4) satisfies

$$
E(t) \leq C E(0) e^{-\alpha t} \quad \text { if } \frac{\rho_{1}}{K}=\frac{\rho_{2}}{b}
$$

Moreover, if the initial value $\mathcal{U}_{0} \in D(\mathcal{A})$, we have that, for some constants $C>0$ and $M_{1}>0$, the energy of problem (2.4) satisfies

$$
E(t) \leq C\left(E(0)+E_{2}(0)\right) t^{-1} \quad \text { if } 0<\left|\frac{\rho_{1}}{K}-\frac{\rho_{2}}{b}\right|<\frac{M_{1} \gamma K}{4(K+b)} .
$$

In order to prove this result, we introduce various functionals and establish several lemmas. The construction of the auxiliary function $I_{1}(t)-I_{3}(t), I_{5}(t)$ comes from [16].

Lemma 4.2 Let $(\phi, \psi, \theta, z)$ be the solution of (2.4). The functional $I_{1}$ defined by

$$
I_{1}(t):=-\int_{0}^{1}\left(\rho_{1} \phi_{t} \phi+\rho_{2} \psi_{t} \psi\right) d x-\frac{\mu_{1}}{2} \int_{0}^{1} \psi^{2} d x
$$

satisfies

$$
\begin{aligned}
I_{1}^{\prime}(t) \leq & -\int_{0}^{1}\left(\rho_{1} \phi_{t}^{2}+\rho_{2} \psi_{t}^{2}\right) d x+\int_{0}^{1} K\left(\phi_{x}+\psi\right)^{2} d x+\left(b+C_{1}+2\right) \int_{0}^{1} \psi_{x}^{2} d x \\
& +\frac{\beta^{2}}{4} \int_{0}^{1} \theta_{t x}^{2} d x+\frac{\mu_{2}^{2}}{4} \int_{0}^{1} z^{2}(x, 1, t) d x .
\end{aligned}
$$


Proof By differentiating $I_{1}$ and using (2.4), we conclude that

$$
\begin{aligned}
I_{1}^{\prime}(t)= & -\int_{0}^{1}\left(\rho_{1} \phi_{t}^{2}+\rho_{2} \psi_{t}^{2}\right) d x+\int_{0}^{1} K\left(\phi_{x}+\psi\right)^{2} d x+b \int_{0}^{1} \psi_{x}^{2} d x+\int_{0}^{1} f(\psi) \psi d x \\
& +\beta \int_{0}^{1} \theta_{t x} \psi d x+\mu_{2} \int_{0}^{1} z(x, 1, t) \psi d x .
\end{aligned}
$$

By using Young's inequality and the fact $\int_{0}^{1} \psi^{2} d x \leq \int_{0}^{1} \psi_{x}^{2} d x$, we have

$$
\begin{aligned}
& \beta \int_{0}^{1} \theta_{t x} \psi d x \leq \frac{\beta^{2}}{4} \int_{0}^{1} \theta_{t x}^{2} d x+\int_{0}^{1} \psi_{x}^{2} d x \\
& \mu_{2} \int_{0}^{1} z(x, 1, t) \psi d x \leq \frac{\mu_{2}^{2}}{4} \int_{0}^{1} z^{2}(x, 1, t) d x+\int_{0}^{1} \psi_{x}^{2} d x .
\end{aligned}
$$

For the fourth term in (4.2), using (2.2) and the generalized Hölder inequality, we obtain

$$
\int_{0}^{1}|f(\psi) \psi| d x \leq k_{0} \int_{0}^{1}|\psi|^{\varsigma}|\psi||\psi| d x \leq k_{0}\|\psi\|_{2(\varsigma+1)}^{\varsigma}\|\psi\|_{2(\varsigma+1)}\|\psi\| .
$$

By Sobolev-Poincaré inequality and $\dot{E}(t) \leq 0$, we get

$$
\|\psi\|_{2(\varsigma+1)} \leq C\left\|\psi_{x}\right\| \leq C\left(\frac{2}{b \gamma} E(t)\right)^{\frac{1}{2}} \leq C\left(\frac{2}{b \gamma}\right)^{\frac{1}{2}} E(0)^{\frac{1}{2}}
$$

in which $C>0$ is a constant. Thus, together with the above two inequalities, Young's inequality and the Sobolev embedding theorem for $\psi$, we obtain

$$
\int_{0}^{1}|f(\psi) \psi| d x \leq C_{1} \int_{0}^{1} \psi_{x}^{2} d x
$$

Insert (4.3), (4.4), and (4.6) into (4.3), then Lemma 4.2 follows.

Lemma 4.3 Let $(\phi, \psi, \theta, z)$ be the solution of (2.4). The functional $I_{2}$ defined by

$$
I_{2}(t):=\int_{0}^{1}\left(\rho_{2} \psi_{t} \psi+\rho_{1} \phi_{t} g\right) d x+\frac{\mu_{1}}{2} \int_{0}^{1} \psi^{2} d x
$$

where $g$ is the solution of

$$
\left\{\begin{array}{l}
-g_{x x}=\psi_{x}, \quad 0<x<1 \\
g(0)=g(1)=0
\end{array}\right.
$$

satisfies that, for any $\varepsilon_{2}>0$,

$$
\begin{aligned}
I_{2}^{\prime}(t) \leq & \left(-b+2 \varepsilon_{2}\right) \int_{0}^{1} \psi_{x}^{2} d x+\rho_{1} \varepsilon_{2} \int_{0}^{1} \phi_{t}^{2} d x+\left(\rho_{2}+\frac{\rho_{1}}{4 \varepsilon_{2}}\right) \int_{0}^{1} \psi_{t}^{2} d x \\
& +\frac{\beta^{2}}{4 \varepsilon_{2}} \int_{0}^{1} \theta_{t x}^{2} d x+\frac{\mu_{2}^{2}}{4 \varepsilon_{2}} \int_{0}^{1} z^{2}(x, 1, t) d x-\int_{0}^{1} \hat{f}(\psi(t)) d x .
\end{aligned}
$$


Proof Using (2.4) and integration by parts, we conclude that

$$
\begin{aligned}
I_{2}^{\prime}(t)= & -b \int_{0}^{1} \psi_{x}^{2} d x+\rho_{2} \int_{0}^{1} \psi_{t}^{2} d x-\beta \int_{0}^{1} \theta_{t x} \psi d x-\mu_{2} \int_{0}^{1} z(x, 1, t) \psi d x \\
& -\int_{0}^{1} f(\psi) \psi d x+\rho_{1} \int_{0}^{1} \varphi_{t} g_{t} d x-K \int_{0}^{1} \psi^{2} d x+K \int_{0}^{1} g_{x}^{2} d x .
\end{aligned}
$$

By the fact following from (4.8) that

$$
\begin{aligned}
& \int_{0}^{1} g_{x}^{2} d x \leq \int_{0}^{1} \psi^{2} d x \leq \int_{0}^{1} \psi_{x}^{2} d x \\
& \int_{0}^{1} g_{t}^{2} d x \leq \int_{0}^{1} g_{x t}^{2} d x \leq \int_{0}^{1} \psi_{t}^{2} d x
\end{aligned}
$$

we obtain that

$$
\begin{aligned}
I_{2}^{\prime}(t) \leq & -b \int_{0}^{1} \psi_{x}^{2} d x+\rho_{2} \int_{0}^{1} \psi_{t}^{2} d x-\beta \int_{0}^{1} \theta_{t x} \psi d x-\mu_{2} \int_{0}^{1} z(x, 1, t) \psi d x \\
& -\int_{0}^{1} \hat{f}(\psi(t)) d x+\rho_{1} \int_{0}^{1} \varphi_{t} g_{t} d x .
\end{aligned}
$$

By using Young's inequality and (4.11)-(4.12), for any $\varepsilon_{2}>0$, we have

$$
\begin{gathered}
\beta \int_{0}^{1} \theta_{t x} \psi d x \leq \frac{\beta^{2}}{4 \varepsilon_{2}} \int_{0}^{1} \theta_{t x}^{2} d x+\varepsilon_{2} \int_{0}^{1} \psi_{x}^{2} d x \\
\mu_{2} \int_{0}^{1} z(x, 1, t) \psi d x \leq \frac{\mu_{2}^{2}}{4 \varepsilon_{2}} \int_{0}^{1} z^{2}(x, 1, t) d x+\varepsilon_{2} \int_{0}^{1} \psi_{x}^{2} d x \\
\rho_{1} \int_{0}^{1} \varphi_{t} g_{t} d x \leq \rho_{1} \varepsilon_{2} \int_{0}^{1} \phi_{t}^{2} d x+\frac{\rho_{1}}{4 \varepsilon_{2}} \int_{0}^{1} g_{t}^{2} d x \\
\leq \rho_{1} \varepsilon_{2} \int_{0}^{1} \phi_{t}^{2} d x+\frac{\rho_{1}}{4 \varepsilon_{2}} \int_{0}^{1} \psi_{t}^{2} d x
\end{gathered}
$$

Combining (4.13)-(4.16), we have (4.9).

Lemma 4.4 Let $(\phi, \psi, \theta, z)$ be the solution of $(2.4)$. The functional $I_{3}$ defined by

$$
I_{3}(t):=\rho_{2} \int_{0}^{1} \psi_{t}\left(\phi_{x}+\psi\right) d x+\rho_{2} \int_{0}^{1} \psi_{x} \phi_{t} d x
$$

satisfies for any $\varepsilon_{3}>0$ that

$$
\begin{aligned}
I_{3}^{\prime}(t) \leq & \left.b \phi_{x} \psi_{x}\right|_{x=0} ^{x=1}+\left(\frac{\rho_{2} K}{\rho_{1}}-b\right) \int_{0}^{1}\left(\phi_{x}+\psi\right)_{x} \psi_{x} d x-\left(\frac{K}{4}-\frac{\varepsilon_{3} C_{2}}{b^{2}}\right) \int_{0}^{1}\left(\phi_{x}+\psi\right)^{2} d x \\
& +\left(\rho_{2}+\frac{\mu_{1}^{2}}{K}\right) \int_{0}^{1} \psi_{t}^{2} d x+\frac{\beta^{2}}{K} \int_{0}^{1} \theta_{t x}^{2} d x+\frac{\mu_{2}^{2}}{K} \int_{0}^{1} z^{2}(x, 1, t) d x \\
& -\int_{0}^{1} \hat{f}(\psi) d x+\left(\frac{\varepsilon_{3} C_{2}}{b^{2}}+\frac{b^{2}}{2 \varepsilon_{3}}\right) \int_{0}^{1} \psi_{x}^{2} d x
\end{aligned}
$$


Proof By differentiating $I_{3}$ and using (2.4), we conclude that

$$
\begin{aligned}
I_{3}^{\prime}(t)= & \left.b \phi_{x} \psi_{x}\right|_{x=0} ^{x=1}+\left(\frac{\rho_{2} K}{\rho_{1}}-b\right) \int_{0}^{1}\left(\phi_{x}+\psi\right)_{x} \psi_{x} d x \\
& -K \int_{0}^{1}\left(\phi_{x}+\psi\right)^{2} d x+\rho_{2} \int_{0}^{1} \psi_{t}^{2} d x \\
& -\beta \int_{0}^{1} \theta_{t x}\left(\phi_{x}+\psi\right) d x-\mu_{1} \int_{0}^{1} \psi_{t}\left(\phi_{x}+\psi\right) d x-\mu_{2} \int_{0}^{1} z(x, 1, t)\left(\phi_{x}+\psi\right) d x \\
& -\int_{0}^{1} f(\psi) \psi d x-\int_{0}^{1} f(\psi) \phi_{x} d x .
\end{aligned}
$$

By using Young's inequality and Poincaré's inequality, we have

$$
\begin{aligned}
& -\beta \int_{0}^{1} \theta_{t x}\left(\phi_{x}+\psi\right) d x \leq \frac{K}{4} \int_{0}^{1}\left(\phi_{x}+\psi\right)^{2} d x+\frac{\beta^{2}}{K} \int_{0}^{1} \theta_{t x}^{2} d x \\
& -\mu_{1} \int_{0}^{1} \psi_{t}\left(\phi_{x}+\psi\right) d x \leq \frac{K}{4} \int_{0}^{1}\left(\phi_{x}+\psi\right)^{2} d x+\frac{\mu_{1}^{2}}{K} \int_{0}^{1} \psi_{t}^{2} d x \\
& -\mu_{2} \int_{0}^{1} z(x, 1, t)\left(\phi_{x}+\psi\right) d x \leq \frac{K}{4} \int_{0}^{1}\left(\phi_{x}+\psi\right)^{2} d x+\frac{\mu_{2}^{2}}{K} \int_{0}^{1} z^{2}(x, 1, t) d x
\end{aligned}
$$

By using the fact that

$$
\int_{0}^{1} \phi_{x}^{2} d x \leq 2 \int_{0}^{1}\left(\phi_{x}+\psi\right)^{2} d x+2 \int_{0}^{1} \psi_{x}^{2} d x
$$

we arrive at, for any $\varepsilon_{3}>0$,

$$
\begin{aligned}
\int_{0}^{1}\left|f(\psi) \phi_{x}\right| d x & \leq k_{0}\|\psi\|_{2(\varsigma+1)}^{\varsigma}\|\psi\|_{2(\varsigma+1)}\left\|\phi_{x}\right\| \\
& \leq \frac{\varepsilon_{3} C_{2}}{2 b^{2}} \int_{0}^{1} \phi_{x}^{2} d x+\frac{b^{2}}{2 \varepsilon_{3}} \int_{0}^{1} \psi_{x}^{2} d x \\
& \leq \frac{\varepsilon_{3} C_{2}}{b^{2}} \int_{0}^{1}\left(\phi_{x}+\psi\right)^{2} d x+\frac{\varepsilon_{3} C_{2}}{b^{2}} \int_{0}^{1} \psi^{2} d x+\frac{b^{2}}{2 \varepsilon_{3}} \int_{0}^{1} \psi_{x}^{2} d x \\
& \leq \frac{\varepsilon_{3} C_{2}}{b^{2}} \int_{0}^{1}\left(\phi_{x}+\psi\right)^{2} d x+\left(\frac{\varepsilon_{3} C_{2}}{b^{2}}+\frac{b^{2}}{2 \varepsilon_{3}}\right) \int_{0}^{1} \psi_{x}^{2} d x
\end{aligned}
$$

in which $C_{2}$ is a positive constant. Combining (4.20)-(4.24) yields the conclusion.

Next we deal with the boundary term in (4.18). We introduce the function

$$
q(x)=-4 x+2, \quad x \in(0,1) .
$$


Lemma 4.5 Let $(\phi, \psi, \theta, z)$ be the solution of (2.4), then for $\varepsilon_{3}>0$ the following estimate holds:

$$
\begin{aligned}
\left.b \phi_{x} \psi_{x}\right|_{x=0} ^{x=1} \leq & -\frac{b \rho_{2}}{4 \varepsilon_{3}} \frac{d}{d t} \int_{0}^{1} q \psi_{t} \psi_{x} d x-\frac{\varepsilon_{3} \rho_{1}}{K} \frac{d}{d t} \int_{0}^{1} q \phi_{t} \phi_{x} d x \\
& +\left(7 \varepsilon_{3}+\frac{b^{2}}{2 \varepsilon_{3}}+\frac{b^{2}}{4 \varepsilon_{3}^{3}}+\frac{3 b^{2}}{4}+\frac{C_{3}}{4 \varepsilon_{3}}+\frac{b}{4 \varepsilon_{3}^{2}}\right) \int_{0}^{1} \psi_{x}^{2} d x \\
& +\left(\frac{\mu_{1}^{2}}{4 \varepsilon_{3}^{2}}+\frac{\rho_{2} b}{2 \varepsilon_{3}}\right) \int_{0}^{1} \psi_{t}^{2} d x+\left(\frac{1}{4} K^{2} \varepsilon_{3}+6 \varepsilon_{3}\right) \int_{0}^{1}\left(\phi_{x}+\psi\right)^{2} d x \\
& +\frac{2 \rho_{1} \varepsilon_{3}}{K} \int_{0}^{1} \phi_{t}^{2} d x+\frac{\beta^{2}}{4 \varepsilon_{3}^{2}} \int_{0}^{1} \theta_{t x}^{2} d x+\frac{\mu_{2}^{2}}{4 \varepsilon_{3}^{2}} \int_{0}^{1} z^{2}(x, 1, t) d x .
\end{aligned}
$$

Proof By using Young's inequality, for $\varepsilon_{3}>0$, we have

$$
\left.b \phi_{x} \psi_{x}\right|_{x=0} ^{x=1} \leq \frac{b^{2}}{4 \varepsilon_{3}}\left[\psi_{x}^{2}(1)+\psi_{x}^{2}(0)\right]+\varepsilon_{3}\left[\phi_{x}^{2}(1)+\phi_{x}^{2}(0)\right] .
$$

Also, we have

$$
\begin{aligned}
\frac{d}{d t} \int_{0}^{1} b \rho_{2} q \psi_{t} \psi_{x} d x= & \left.\frac{1}{2} b^{2} q \psi_{x}^{2}\right|_{x=0} ^{x=1}-\frac{1}{2} \int_{0}^{1} b^{2} q_{x} \psi_{x}^{2} d x-\frac{1}{2} b \rho_{2} \int_{0}^{1} q_{x} \psi_{t}^{2} d x \\
& -b K \int_{0}^{1} q\left(\phi_{x}+\psi\right) \psi_{x} d x-b \beta \int_{0}^{1} q \theta_{t x} \psi_{x} d x \\
& -\mu_{1} b \int_{0}^{1} q \psi_{t} \psi_{x} d x-\mu_{2} b \int_{0}^{1} q z(x, 1, t) \psi_{x} d x \\
& -b \int_{0}^{1} q f(\psi) \psi_{x} d x .
\end{aligned}
$$

By using Young's inequality and Poincaré's inequality, for $\varepsilon_{3}>0$, we have

$$
\begin{aligned}
\frac{d}{d t} \int_{0}^{1} b \rho_{2} q \psi_{t} \psi_{x} d x \leq & -b^{2}\left[\psi_{x}^{2}(1)+\psi_{x}^{2}(0)\right] \\
& +\left(2 b^{2}+\frac{b^{2}}{\varepsilon_{3}^{2}}+3 \varepsilon_{3} b^{2}+\frac{b}{\varepsilon_{3}}+C_{3}\right) \int_{0}^{1} \psi_{x}^{2} d x \\
& +\left(2 \rho_{2} b+\frac{\mu_{1}^{2}}{\varepsilon_{3}}\right) \int_{0}^{1} \psi_{t}^{2} d x+K^{2} \varepsilon_{3}^{2} \int_{0}^{1}\left(\phi_{x}+\psi\right)^{2} d x \\
& +\frac{\beta^{2}}{\varepsilon_{3}} \int_{0}^{1} \theta_{t x}^{2} d x+\frac{\mu_{2}^{2}}{\varepsilon_{3}} \int_{0}^{1} z^{2}(x, 1, t) d x
\end{aligned}
$$

Similarly,

$$
\begin{aligned}
\frac{d}{d t} \int_{0}^{1} \rho_{1} q \phi_{t} \phi_{x} d x \leq & -K\left[\phi_{x}^{2}(1)+\phi_{x}^{2}(0)\right] \\
& +3 K \int_{0}^{1} \phi_{x}^{2} d x+K \int_{0}^{1} \psi_{x}^{2} d x+2 \rho_{1} \int_{0}^{1} \phi_{t}^{2} d x
\end{aligned}
$$

Together with (4.27)-(4.29), using (4.23) gives us (4.26). 
Lemma 4.6 Let $(\phi, \psi, \theta, z)$ be the solution of (2.4). The functional $I_{4}$ defined by

$$
I_{4}(t):=\int_{0}^{1}\left(\rho_{3} \theta_{t} \theta+\frac{k}{2} \theta_{x}^{2}+\gamma \psi_{x} \theta\right) d x
$$

and its time derivative $I_{4}^{\prime}(t)$ satisfies

$$
I_{4}^{\prime}(t) \leq-\delta \int_{0}^{1} \theta_{x}^{2} d x+\int_{0}^{1} \psi_{x}^{2} d x+C_{4}\left(\frac{\gamma^{2}}{4}+\rho_{3}\right) \int_{0}^{1} \theta_{t x}^{2} d x
$$

where $C_{4}>0$ is the Sobolev embedding constant.

Proof By differentiating $I_{4}$ and using (2.4), we conclude that

$$
I_{4}^{\prime}(t)=-\delta \int_{0}^{1} \theta_{x}^{2} d x+\rho_{3} \int_{0}^{1} \theta_{t}^{2} d x+\gamma \int_{0}^{1} \psi_{x} \theta_{t} d x
$$

Using Young's inequality and Poincaré's inequality clearly implies the conclusion (4.30).

Lemma 4.7 Let $(\phi, \psi, \theta, z)$ be the solution of (2.4). The functional $I_{5}$ defined by

$$
I_{5}(t):=\int_{0}^{1} \int_{0}^{1} e^{-2 \tau \rho} z^{2}(x, \rho, t) d \rho d x
$$

for some constant $m>0$ satisfies

$$
I_{5}^{\prime}(t) \leq-m \int_{0}^{1} z^{2}(x, 1, t) d x-m \int_{0}^{1} \int_{0}^{1} z^{2}(x, \rho, t) d \rho d x+\frac{1}{\tau} \int_{0}^{1} \psi_{t}^{2} d x .
$$

Proof By differentiating $I_{5}$ and using (2.4), we conclude that

$$
\begin{aligned}
I_{5}^{\prime}(t) & =-\frac{2}{\tau} \int_{0}^{1} \int_{0}^{1} e^{-2 \tau \rho} z z_{\rho}(x, \rho, t) d \rho d x=-\frac{1}{\tau} \int_{0}^{1} \int_{0}^{1} e^{-2 \tau \rho} \frac{\partial}{\partial \rho} z^{2}(x, \rho, t) d \rho d x \\
& =-2 \int_{0}^{1} \int_{0}^{1} e^{-2 \tau \rho} z^{2}(x, \rho, t) d \rho d x-\frac{1}{\tau} \int_{0}^{1} \int_{0}^{1} \frac{\partial}{\partial \rho}\left(e^{-2 \tau \rho} z^{2}(x, \rho, t)\right) d \rho d x \\
& \leq-m \int_{0}^{1} z^{2}(x, 1, t) d x-m \int_{0}^{1} \int_{0}^{1} z^{2}(x, \rho, t) d \rho d x+\frac{1}{\tau} \int_{0}^{1} \psi_{t}^{2} d x .
\end{aligned}
$$

This gives (4.31).

Now we define the Lyapunov functional $\mathscr{L}(t)$ as follows:

$$
\begin{aligned}
\mathcal{L}(t):= & N E(t)+\frac{1}{8} I_{1}(t)+N_{2} I_{2}(t)+I_{3}(t)+I_{4}(t)+I_{5}(t) \\
& +\frac{b \rho_{2}}{4 \varepsilon_{3}} \int_{0}^{1} q \psi_{t} \psi_{x} d x+\frac{\varepsilon_{3} \rho_{1}}{K} \int_{0}^{1} q \phi_{t} \phi_{x} d x,
\end{aligned}
$$

where $N, N_{2}$ are positive constants to be chosen properly later. For $N$ large enough, it is not difficult to prove that there exist two positive constants $\gamma_{1}$ and $\gamma_{2}$ such that, for any $t>0$,

$$
\gamma_{1} E(t) \leq \mathscr{L}(t) \leq \gamma_{2} E(t)
$$


Proof of Theorem 4.1 Combining Lemmas 4.2-4.7, we have

$$
\begin{aligned}
\mathcal{L}^{\prime}(t) \leq & \left(-C N+\frac{\beta^{2}}{32}+N_{2} \frac{\beta^{2}}{4 \varepsilon_{2}}+\frac{\beta^{2}}{K}+\frac{\beta^{2}}{4 \varepsilon_{3}^{2}}+C_{4}\left(\frac{\gamma^{2}}{4}+\rho_{3}\right)\right) \int_{0}^{1} \theta_{t x}^{2} d x \\
& +\left(-C N-\frac{1}{8} \rho_{2}+N_{2}\left(\frac{\rho_{1}}{4 \varepsilon_{2}}+\rho_{2}\right)+\rho_{2}+\frac{\mu_{1}^{2}}{K}+\frac{\mu_{1}^{2}}{4 \varepsilon_{3}^{2}}+\frac{\rho_{2} b}{2 \varepsilon_{3}}+\frac{1}{\tau}\right) \int_{0}^{1} \psi_{t}^{2} d x \\
& +\left(-C N-m+\frac{\mu_{2}^{2}}{32}+N_{2} \frac{\mu_{2}^{2}}{4 \varepsilon_{2}}+\frac{\mu_{2}^{2}}{K}+\frac{\mu_{2}^{2}}{4 \varepsilon_{3}^{2}}\right) \int_{0}^{1} z^{2}(x, 1, t) d x \\
& +\left(-\frac{1}{8} \rho_{1}+N_{2} \rho_{1} \varepsilon_{2}+\frac{2 \rho_{1} \varepsilon_{3}}{K}\right) \int_{0}^{1} \phi_{t}^{2} d x+\left[N_{2}\left(-b+2 \varepsilon_{2}\right)+\frac{1}{8}\left(b+C_{1}+2\right)\right. \\
& \left.+7 \varepsilon_{3}+\frac{b^{2}}{2 \varepsilon_{3}}+\frac{b^{2}}{4 \varepsilon_{3}^{3}}+\frac{3 b^{2}}{4}+\frac{C_{3}}{4 \varepsilon_{3}}+\frac{b}{4 \varepsilon_{3}^{2}}+\frac{\varepsilon_{3} C_{2}}{b^{2}}+\frac{b^{2}}{2 \varepsilon_{3}}+1\right] \int_{0}^{1} \psi_{x}^{2} d x \\
& +\left(-\frac{1}{8} K+\frac{1}{4} K^{2} \varepsilon_{3}+6 \varepsilon_{3}+\frac{\varepsilon_{3} C_{2}}{b^{2}}\right) \int_{0}^{1}\left(\phi_{x}+\psi\right)^{2} d x \\
& +(-\delta) \int_{0}^{1} \theta_{x}^{2} d x+(-m) \int_{0}^{1} \int_{0}^{1} z^{2}(x, \rho, t) d \rho d x+\left(-N_{2}-1\right) \int_{0}^{1} \hat{f}(\psi) d x \\
& +\left(\frac{\rho_{2} K}{\rho_{1}}-b\right) \int_{0}^{1}\left(\phi_{x}+\psi\right)_{x} \psi_{x} d x .
\end{aligned}
$$

Firstly, we take $\varepsilon_{3}$ small enough such that

$$
\left\{\begin{array}{l}
-\frac{1}{8} K+\frac{1}{4} K^{2} \varepsilon_{3}+6 \varepsilon_{3}+\frac{\varepsilon_{3} C_{2}}{b^{2}}<0 \\
-\frac{1}{8}+\frac{2 \varepsilon_{3}}{K}<0
\end{array}\right.
$$

Then we choose $N_{2}$ so large that

$$
N_{2} b>2\left[\frac{1}{8}\left(b+C_{1}+2\right)+7 \varepsilon_{3}+\frac{b^{2}}{2 \varepsilon_{3}}+\frac{b^{2}}{4 \varepsilon_{3}^{3}}+\frac{3 b^{2}}{4}+\frac{C_{3}}{4 \varepsilon_{3}}+\frac{b}{4 \varepsilon_{3}^{2}}+\frac{\varepsilon_{3} C_{2}}{b^{2}}+\frac{b^{2}}{2 \varepsilon_{3}}+1\right]=: \Xi,
$$

thus we have

$$
-N_{2} b+\frac{1}{2} \Xi<-\frac{1}{2} \Xi
$$

After that, we select $\varepsilon_{2}$ small enough such that

$$
-\frac{1}{2} \Xi+N_{2} \varepsilon_{2}<0
$$

and

$$
-\frac{1}{8}+\frac{2 \varepsilon_{3}}{K}+N_{2} \varepsilon_{2}<0
$$

Finally, we choose $N$ so large that

$$
-C N+\frac{\beta^{2}}{32}+N_{2} \frac{\beta^{2}}{4 \varepsilon_{2}}+\frac{\beta^{2}}{K}+\frac{\beta^{2}}{4 \varepsilon_{3}^{2}}+C_{4}\left(\frac{\gamma^{2}}{4}+\rho_{3}\right)<0
$$




$$
\begin{aligned}
& -C N-\frac{1}{8} \rho_{2}+N_{2}\left(\frac{\rho_{1}}{4 \varepsilon_{2}}+\rho_{2}\right)+\rho_{2}+\frac{\mu_{1}^{2}}{K}+\frac{\mu_{1}^{2}}{4 \varepsilon_{3}^{2}}+\frac{\rho_{2} b}{2 \varepsilon_{3}}+\frac{1}{\tau}<0, \\
& -C N-m+\frac{\mu_{2}^{2}}{32}+N_{2} \frac{\mu_{2}^{2}}{4 \varepsilon_{2}}+\frac{\mu_{2}^{2}}{K}+\frac{\mu_{2}^{2}}{4 \varepsilon_{3}^{2}}<0 .
\end{aligned}
$$

Therefore (4.34) changes to

$$
\begin{aligned}
\mathcal{L}^{\prime}(t) \leq & -M \int_{0}^{1}\left(\theta_{t x}^{2}+\psi_{t}^{2}+z^{2}(x, 1, t)+\phi_{t}^{2}+\psi_{x}^{2}+\left(\phi_{x}+\psi\right)^{2}+\theta_{x}^{2}\right) d x \\
& -M \int_{0}^{1} \int_{0}^{1} z^{2}(x, \rho, t) d \rho d x+\left(\frac{\rho_{2} K}{\rho_{1}}-b\right) \int_{0}^{1}\left(\phi_{x}+\psi\right)_{x} \psi_{x} d x \\
\leq & -M_{1} E(t)+\left(\frac{\rho_{2} K}{\rho_{1}}-b\right) \int_{0}^{1}\left(\phi_{x}+\psi\right)_{x} \psi_{x} d x
\end{aligned}
$$

where $M, M_{1}$ are positive constants.

Case 1: $\frac{\rho_{1}}{K}=\frac{\rho_{2}}{b}$.

In this case, (4.35) takes the form

$$
\mathcal{L}^{\prime}(t) \leq-M_{1} E(t)
$$

Using (4.33), we get, for $\alpha=\frac{M_{1}}{\gamma_{2}}$,

$$
\mathcal{L}^{\prime}(t) \leq-\alpha \mathcal{L}(t)
$$

A simple integration of $(4.36)$ over $(0, t)$ leads to

$$
\mathcal{L}(t) \leq \mathcal{L}(0) e^{-\alpha t}
$$

Recalling (4.33), we obtain

$$
E(t) \leq C E(0) e^{-\alpha t}
$$

Case 2: $0<\left|\frac{\rho_{1}}{K}-\frac{\rho_{2}}{b}\right|<\frac{M_{1} \gamma K}{4(K+b)}$.

Let

$$
E_{1}(t):=E(t)
$$

represent the first order energy defined in (3.6). By computation we have the estimate of the derivative of the second order energy (3.7) as

$$
E_{2}^{\prime}(t) \leq-C \int_{0}^{1}\left(\theta_{t t x}^{2}+\psi_{t t}^{2}+z_{t}^{2}(x, 1, t)\right) d x
$$


Hoo and Wei Boundary Value Problems (2018) 2018:65

Page 15 of 17

Let us estimate the last term in (4.35). By setting $\Lambda=\frac{1}{K}\left(\frac{\rho_{2} K}{\rho_{1}}-b\right) \rho_{1} \neq 0$ and using (2.4), (4.23), we have

$$
\begin{aligned}
\left(\frac{\rho_{2} K}{\rho_{1}}-b\right) \int_{0}^{1}\left(\phi_{x}+\psi\right)_{x} \psi_{x} d x= & \frac{1}{K}\left(\frac{\rho_{2} K}{\rho_{1}}-b\right) \int_{0}^{1} \rho_{1} \phi_{t t} \psi_{x} d x \\
= & \Lambda \int_{0}^{1} \phi_{t t} \psi_{x} d x \\
= & -\Lambda\left(\frac{d}{d t} \int_{0}^{1} \phi_{x t} \psi d x-\frac{d}{d t} \int_{0}^{1} \phi_{x} \psi_{t} d x\right) \\
& -\Lambda \int_{0}^{1} \phi_{x} \psi_{t t} d x \\
\leq & -\Lambda\left(\frac{d}{d t} \int_{0}^{1} \phi_{x t} \psi d x-\frac{d}{d t} \int_{0}^{1} \phi_{x} \psi_{t} d x\right) \\
& +\frac{|\Lambda|}{4} \int_{0}^{1} \psi_{t t}^{2} d x \\
& +2|\Lambda| \int_{0}^{1}\left(\phi_{x}+\psi\right)^{2} d x+2|\Lambda| \int_{0}^{1} \psi_{x}^{2} d x
\end{aligned}
$$

Let

$$
\mathcal{N}(t):=\int_{0}^{1} \phi_{x t} \psi d x-\int_{0}^{1} \phi_{x} \psi_{t} d x
$$

Then (4.35) becomes

$$
\mathcal{L}^{\prime}(t)+\Lambda \mathcal{N}^{\prime}(t) \leq-M_{2} E_{1}(t)+\frac{|\Lambda|}{4} \int_{0}^{1} \psi_{t t}^{2} d x
$$

for $M_{2}=M_{1}-\frac{4|\Lambda|}{\gamma}\left(\frac{1}{K}+\frac{1}{b}\right)>0$. Let

$$
F(t)=\mathcal{L}(t)+\Lambda \mathcal{N}(t)+N_{3}\left(E_{1}(t)+E_{2}(t)\right) \geq 0
$$

if $N_{3}>\max \left\{C_{0}|\Lambda|-\gamma_{1},|\Lambda|, \frac{|\Lambda|}{4 C}\right\}$. Indeed, by using (4.11), (4.23), and $a b \leq \frac{1}{2} a^{2}+\frac{1}{2} b^{2}$, we obtain

$$
\begin{aligned}
|\mathcal{N}(t)| & \leq\left|\int_{0}^{1} \phi_{x t} \psi d x\right|+\left|\int_{0}^{1} \phi_{x} \psi_{t} d x\right| \\
& \leq \frac{1}{2} \int_{0}^{1} \phi_{x t}^{2} d x+\frac{1}{2} \int_{0}^{1} \psi^{2} d x+\frac{1}{2} \int_{0}^{1} \phi_{x}^{2} d x+\frac{1}{2} \int_{0}^{1} \psi_{t}^{2} d x \\
& \leq \frac{1}{2} \int_{0}^{1} \phi_{x t}^{2} d x+\frac{1}{2} \int_{0}^{1} \psi_{x}^{2} d x+\int_{0}^{1}\left(\phi_{x}+\psi\right)^{2} d x+\int_{0}^{1} \psi_{x}^{2} d x+\frac{1}{2} \int_{0}^{1} \psi_{t}^{2} d x \\
& \leq E_{2}(t)+\max \left\{\frac{3}{b \gamma}, \frac{2}{K \gamma}, \frac{1}{\rho_{2} \gamma}\right\} E_{1}(t):=E_{2}(t)+C_{0} E_{1}(t),
\end{aligned}
$$


where $C_{0}=\max \left\{\frac{3}{b \gamma}, \frac{2}{K \gamma}, \frac{1}{\rho_{2} \gamma}\right\}$. With the help of (4.33), we obtain

$$
\begin{aligned}
F(t) & \geq \gamma_{1} E_{1}(t)-|\Lambda|\left(E_{2}(t)+C_{0} E_{1}(t)\right)+N_{3}\left(E_{1}(t)+E_{2}(t)\right) \\
& \geq\left(N_{3}+\gamma_{1}-C_{0}|\Lambda|\right) E_{1}(t)+\left(N_{3}-|\Lambda|\right) E_{2}(t) \geq 0 .
\end{aligned}
$$

It is easy to prove that

$$
c_{1}\left(E_{1}(t)+E_{2}(t)\right) \leq F(t) \leq c_{2}\left(E_{1}(t)+E_{2}(t)\right)
$$

for some positive constants $c_{1}$ and $c_{2}$. By using (4.39) and (4.40), we obtain

$$
\begin{aligned}
F^{\prime}(t) & =\mathcal{L}^{\prime}(t)+\Lambda \mathcal{N}^{\prime}(t)+N_{3}\left(E_{1}^{\prime}(t)+E_{2}^{\prime}(t)\right) \\
& \leq-M_{2} E_{1}(t)+\left(-C N_{3}+\frac{|\Lambda|}{4}\right) \int_{0}^{1} \psi_{t t}^{2} d x
\end{aligned}
$$

Thanks to the choice of $N_{3}$, we have

$$
F^{\prime}(t) \leq-M_{2} E_{1}(t)
$$

Integrating (4.43) over $(0, t)$ yields

$$
\int_{0}^{t} E_{1}(r) d r \leq \frac{1}{M_{2}}(F(0)-F(t)) \leq \frac{1}{M_{2}} F(0) \leq \frac{c_{2}}{M_{2}}\left(E_{1}(0)+E_{2}(0)\right) .
$$

Using the fact that

$$
\left(t E_{1}(t)\right)^{\prime}=t E_{1}^{\prime}(t)+E_{1}(t) \leq E_{1}(t),
$$

we get that

$$
t E_{1}(t) \leq \frac{c_{2}}{M_{2}}\left(E_{1}(0)+E_{2}(0)\right) .
$$

This completes the proof of Theorem 4.1.

Acknowledgements

The authors cordially thank the anonymous referees for their valuable comments and suggestions which led to the improvement of this paper.

Funding

This work was partially supported by NNSF of China (61374089).

\section{Abbreviations}

Not applicable.

Availability of data and materials

Not applicable.

Ethics approval and consent to participate

Not applicable. 
Authors' contributions

The authors declare that the study was realized in collaboration with the same responsibility. All authors read and approved the final manuscript.

\section{Publisher's Note}

Springer Nature remains neutral with regard to jurisdictional claims in published maps and institutional affiliations.

Received: 4 October 2017 Accepted: 18 April 2018 Published online: 27 April 2018

\section{References}

1. Messaoudi, S.A., Said-Houari, B.: Energy decay in a Timoshenko-type system of thermoelasticity of type III. J. Math. Anal. Appl. 348, 298-307 (2008)

2. Messaoudi, S.A., Fareh, A.: Energy decay in a Timoshenko-type system of thermoelasticity of type III with different wave-propagation speeds. Arab. J. Math. 2, 199-207 (2013)

3. Ayadi, M.A., Bchatnia, A., Hamouda, M., Messaoudi, S.: General decay in a Timoshenko-type system with thermoelasticity with second sound. Adv. Nonlinear Anal. 4, 263-284 (2015)

4. Grace, S.: Oscillation criteria for third order nonlinear delay differential equations with damping. Opusc. Math. 35, 485-497 (2015)

5. Öztürk, Ö., Akmon, E.: Nonoscillatory solutions of two dimensional nonlinear delay dynamical systems. Opusc. Math. 36, 651-669 (2016)

6. Racke, R.: Instability of coupled systems with delay. Commun. Pure Appl. Anal. 11, 1753-1773 (2012)

7. Nicaise, S., Pignotti, C., Valein, J.: Exponential stability of the wave equation with boundary time-varying delay. Discrete Contin. Dyn. Syst., Ser. S 4, 693-722 (2011)

8. Nicaise, S., Pignotti, C.: Stability of the wave equation with boundary or internal distributed delay. Differ. Integral Equ. 21, 935-985 (2008)

9. Apalara, T.A., Messaoudi, S.A.: An exponential stability result of a Timoshenko system with thermoelasticity with second sound and in the presence of delay. Appl. Math. Optim. 71, 449-472 (2015)

10. Guesmia, A., Messaoudi, S.A.: A general stability result in a Timoshenko system with infinite memory: a new approach. Math. Methods Appl. Sci. 37, 384-392 (2014)

11. Apalara, T.A., Messaoudi, S.A.: General stability result in a memory-type porous thermoelasticity system of type III. Arab J. Math. Sci. 20, 213-232 (2014)

12. Messaoudi, S.A., Said-Houari, B.: Uniform decay in a Timoshenko-type system with past history. J. Math. Anal. Appl. 360, 459-475 (2009)

13. Guesmia, A., Messaoudi, S.A.: On the stabilization of Timoshenko systems with memory and different speeds of wave propagation. Appl. Math. Comput. 219, 9424-9437 (2013)

14. Suh, H., Bien, Z.: Use of time delay action in the controller design. IEEE Trans. Autom. Control 25, 600-603 (1980)

15. Kafini, M., Messaoudi, S.A., Mustafa, M.I.: Well-posedness and stability results in a Timoshenko-type system of thermoelasticity of type III with delay. Z. Angew. Math. Phys. 66, 1499-1517 (2015)

16. Feng, B.W., Pelicer, M.L.: Global existence and exponential stability for a nonlinear Timoshenko system with delay. Bound. Value Probl. 2015, Article ID 206 (2015)

17. Pazy, A.: Semigroups of Linear Operators and Applications to Partial Differential Equations. Springer, New York (1983)

\section{Submit your manuscript to a SpringerOpen ${ }^{\odot}$ journal and benefit from:}

- Convenient online submission

- Rigorous peer review

- Open access: articles freely available online

- High visibility within the field

- Retaining the copyright to your article

Submit your next manuscript at $\boldsymbol{\triangleright}$ springeropen.com 